\title{
Generalized contest success functions
}

\author{
Birendra K. Rai • Rajiv Sarin
}

Received: 23 April 2007 / Accepted: 18 December 2007 / Published online: 12 January 2008 (C) Springer-Verlag 2008

\begin{abstract}
The key element of models of contest is the contest success function (CSF) which specifies the winning probabilities of agents. The existing axiomatizations of CSFs assume that contestants can make only one type of investment. This paper generalizes these axiomatizations to the case where each agent can have multiple types of investments. This allows us to provide a unified framework to extend and interpret the results of Skaperdas (Econ Theory 7:283-290, 1996) and Clark and Riis (Econ Theory 11:201-204, 1998), and rationalize some seemingly ad hoc CSFs used by applied researchers.
\end{abstract}

Keywords Contest · Tournaments · Conflict - Rent Seeking · Axiomatization

JEL Classification $\quad \mathrm{C} 70 \cdot \mathrm{D} 72 \cdot \mathrm{D} 74$

\section{Introduction}

Over the last few decades models of contest have been used to study rent seeking (Nitzan 1994) and conflict (Skaperdas and Garfinkel 2006) by political economists, reward structure in firms (Rosen 1986) by labor economists, electoral competition (Snyder 1989) and design of optimal voluntary contribution mechanisms by public

This paper has benefitted from the comments of Aicke Hinrichs, Rene Levinsky, and two anonymous referees. We are especially thankful to one of the referees for detailed comments which have helped improve every aspect of this paper.

B. K. Rai $(\varangle) \cdot$ R. Sarin

Max Planck Institute of Economics, Kahlaische Strasse 10, Jena 07745, Germany

e-mail: rai@econ.mpg.de

B. K. Rai · R. Sarin

Department of Economics, Texas A\&M University, College Station, TX 77843, USA 
economists (Kolmar and Wagener 2005), and individual and team sports (Szymanzki 2003) by sports economists. A contest is modeled as a non-cooperative game between multiple agents. Agents make irreversible investments, which can be effort, money, or any other valuable resource depending on the context, to increase their probability of winning the contest and obtaining a private prize.

The key element of all models of contest is the contest success function (CSF) which specifies the winning probabilities of the contestants. Skaperdas (1996) axiomatizes several classes of CSFs in which each agent has a single type of investment (for example, effort). Clark and Riis (1998) extend it by allowing contestants to differ in their contest-relevant personal characteristics. ${ }^{1}$ We aim to generalize the axiomatization of Skaperdas (1996) to the case where agents can have multiple types of investments. We think of the various actual investments by an agent as inputs that he combines using his ${ }^{2}$ technology to produce effective investments. The effective investments then determine the winning probabilities. In our framework one can interpret the contestrelevant personal characteristics of a contestant as yet another type of input (which is fixed, and not a choice variable for the contestant). The primary contribution of our paper is thus to provide a unified framework to extend and interpret the results of Skaperdas (1996) and Clark and Riis (1998) by allowing multiple types of investments for each contestant. Consequently, several seemingly ad hoc CSFs that have been used by applied researchers arise as special cases of the family of CSFs axiomatized in this paper. $^{3}$

Section 2 provides a brief discussion of the four basic axioms of probability, consistency, independence of irrelevant alternatives, and marginal effects. Section 3 first axiomatizes the class of CSFs satisfying the four basic axioms and homogeneity of order zero with respect to each investment of each contestant, and then clarifies the impact of the axiom of anonymity. Section 4 concludes. The appendix contains all the proofs.

\section{The axioms}

Let $N$ represent the finite set of contestants $\{1,2, \ldots, i, \ldots, n\}$. We shall denote the vector of investments by contestant- $i$ as $z_{i} \in \mathbb{R}_{+}^{L}$. Some of the $L$ types of investments may not be a choice variable for a contestant during the contest. For example, in a sporting contest the personal talents and abilities of players can be treated as fixed investments (not subject to choice during the contest) that influence the winning probabilities. The probability that contestant- $i$ wins the contest will be denoted as

$$
p_{i}(z)=p_{i}\left(z_{1}, z_{2}, \ldots, z_{i}, \ldots, z_{n}\right), \quad \forall i \in N,
$$

\footnotetext{
${ }^{1}$ They drop the axiom of anonymity which requires agents with equal investments to have equal winning probabilities.

2 We will later distinguish between unmediated and mediated contests depending upon whether contestants possess their own technology to produce effective investments, or it is determined by the contest administrator.

${ }^{3}$ We achieve this without introducing any new axioms. We simply state the axioms of Skaperdas (1996) for the case where each contestant can have multiple types of investments.
} 
where each $p_{i}(z) \in[0,1]$ for all $z \in \mathbb{R}_{+}^{L N}$, and $p_{i}(z)$ is strictly positive if each investment by contestant $i$ is strictly greater than zero. The function $p_{i}(z)$ is the CSF.

We shall refer to the contest involving all the $N$ agents as the global contest, and any contest involving agents in $M \subseteq N$ as a sub-contest. ${ }^{4} p_{i}^{M}(z)$ will henceforth be used to denote the winning probabilities of agents involved in the sub-contest. Similarly, the vector $z^{M} \in \mathbb{R}_{+}^{L M}$ will be used to represent the investment by agents in $M \subseteq N$. For any given $z \in \mathbb{R}_{+}^{L N}$, the four axioms introduced by Skaperdas (1996) are

[A1] Probability $\sum_{i \in N} \quad p_{i}(z)=1$.

[A2] Consistency $p_{i}^{M}(z)=\frac{p_{i}^{N}(z)}{\sum_{k \in M} p_{k}^{N}(z)}, \quad \forall i \in M, \quad$ and $\quad \forall M \subseteq N$.

[A3] IIA $p_{i}^{M}(z)=p_{i}^{M}\left(z^{M}\right), \quad \forall i \in M, \quad$ and $\forall M \subseteq N$, where $z=\left(z^{M}, z^{N \backslash M}\right)$.

[A4] Marginal effects $p_{i}\left(\tilde{z}_{i}, z_{-i}\right)>p_{i}\left(z_{i}, z_{-i}\right)$ if $\tilde{z}_{i}>z_{i}, \quad \forall i \in N$.

The axiom of probability requires the winning probabilities to sum to unity. For a given vector of investments $z \in \mathbb{R}_{+}^{L N}$, consider the sub-contest among agents in $M \subseteq N$. Consistency requires the probability that $i \in M$ wins this $\operatorname{sub}$-contest $\left(p_{i}^{M}(z)\right)$ to be the same as the probability that $i$ would have won the global contest among all the $N$ agents, given that one of the agents in $M$ wins it. Consistency does not, however, rule out the possibility that the winning probability of an agent in $M$ might depend on the investments of agents outside $M$. The axiom of independence of irrelevant alternatives $\left(\right.$ IIA $_{S}$ ) is introduced to eliminate this possibility. The axiom of marginal effects prescribes that the winning probability of a contestant should be increasing in each of his investments.

Theorem 1 The axioms A1-A4 are satisfied if and only if

$$
p_{i}(z)=\frac{f_{i}\left(z_{i}\right)}{\sum_{k \in N} f_{k}\left(z_{k}\right)}, \quad \forall z \in \mathbb{R}_{+}^{L N}, \quad \forall i \in N,
$$

where each $f_{i}(\cdot)$ is increasing in each of its arguments.

Proof In the Appendix.

Theorem 1 essentially says that the winning probability of each agent can be expressed using $n$ functions, where the function corresponding to contestant- $i$ depends only on his own vector of investments, $z_{i} \in \mathbb{R}_{+}^{L}$. We interpret the value of $f_{i}\left(z_{i}\right)$ as the level of effective investment by contestant- $i$. The effective investment of contestant- $i$ is like an output determined by the levels of the $L$ inputs $\left(z_{i}^{1}, z_{i}^{2}, \ldots, z_{i}^{L}\right)$, and the technology of combining these inputs $\left(f_{i}(\cdot)\right)$. The technology of producing effective investments can differ across agents, as reflected by the subscript $i$, since we have not imposed the axiom of anonymity. The ratio of winning probabilities of any two agents is thus equal to the ratio of their effective investments.

\footnotetext{
${ }^{4}$ Suppose we somehow know that the winner of the contest belongs to the set $M$ which is a subset of $N$. The winning probabilities of the contestants in $M$ can be updated given this new information. The concept of a sub-contest is used to propose some reasonable conditions regarding (a) how the updated probability of any contestant in the winning set $M$ should be related to initial probabilities, and (b) what should the updated probabilities (not) depend upon.
} 


\section{Axiomatization}

Skaperdas (1996) considers contests where each agent can make only one type of investment and proves that a CSF satisfies the axioms of probability, consistency, independence of irrelevant alternatives, marginal effects, homogeneity of order zero with respect to investments, and anonymity if and only if it is the anonymous TullockCSF given by

$$
p_{i}(x)=p_{i}\left(x_{1}, \ldots, x_{i}, \ldots, x_{n}\right)=\frac{x_{i}^{\theta}}{\sum_{k \in N} x_{k}^{\theta}}, \quad \forall x \in \mathbb{R}_{+}^{N}, \quad \forall i \in N
$$

where $\theta$ is a strictly positive constant and $x_{i} \in \mathbb{R}_{+}$is the investment by contestant $-i$. Clark and Riis (1998) argue that the anonymity axiom is inappropriate in several contests as the winning probabilities may depend not only on the investments but also on the "personal characteristics" of the contestants. They go on to prove that a CSF satisfies the axioms of probability, IIA $\mathrm{Cr}^{5},{ }^{5}$ marginal effects, and homogeneity with respect to investments if and only if it is the non-anonymous Tullock-CSF given by

$$
p_{i}(x)=\frac{\alpha_{i} x_{i}^{\theta}}{\sum_{k \in N} \alpha_{k} x_{k}^{\theta}}, \quad \forall x \in \mathbb{R}_{+}^{N}, \quad \forall i \in N,
$$

where $\alpha_{i}$ represents the contest-relevant personal characteristics of contestant- $i$. Note that Clark and Riis treat the personal characteristics of contestants as exogenous parameters.

\subsection{Homogeneity}

We now provide an axiomatization for the CSFs that can be used to analyze contests in which agents can have multiple types of investments using the four axioms discussed in the previous section, and the following axiom.

[A5] Homogeneity $p_{i}(\lambda z)=p_{i}(z), \quad \forall \lambda>0, \quad \forall z \in \mathbb{R}_{+}^{L N}, \quad \forall i \in N$.

Homogeneity requires that the winning probabilities should remain unchanged if each type of investment of all the contestants changes by the same multiplicative factor. The main result of the paper follows.

Theorem 2 The axioms A1-A5 are satisfied if and only if

$$
p_{i}(z)=\frac{f_{i}\left(z_{i}\right)}{\sum_{k \in N} f_{k}\left(z_{k}\right)}, \quad \forall z \in \mathbb{R}_{+}^{L N}, \quad \forall i \in N,
$$

\footnotetext{
5 Clark and Riis (1998) propose to combine consistency and IIA $A_{S}$ into IIA $A_{c r}$. However, IIA by only those CSFs that lead to a winning probability of zero for a contestant who invests zero. It can be easily verified that the Logit-CSF satisfies consistency and IIA
} 
where each $f_{i}(\cdot)$ is a homogenous function of the same degree $\mu>0$, and increasing in each of its arguments.

Proof In the Appendix.

Imposing homogeneity in addition to the four basic axioms leads to a family of CSFs characterized by the fact that the production function of each contestant is a homogenous function. Moreover, the degree of homogeneity of these production functions is the same for all contestants. Obviously, it allows the specific form of production functions to vary across contestants.

We now present some examples of CSFs that belong to the family axiomatized in Theorem 2. In all the following examples, without loss of generality, we shall restrict ourselves to the case with only two types of investments. We shall denote the investment vector of contestant $i$ by $z_{i}=\left(x_{i}, y_{i}\right)$. The overall investment vector will be denoted by $z=(x, y)$.

Example $1 f_{i}\left(x_{i}, y_{i}\right)=\left(\theta_{i} \cdot x_{i}+y_{i}\right)$, where $\theta_{i}>0$, for all $i \in N$.

This example represents the case where each agent has a linear production function for effective investment, but the marginal rate of technical substitution among the two investments varies across the contestants. We will obtain

$$
p_{i}(x, y)=\frac{\left(\theta_{i} \cdot x_{i}+y_{i}\right)}{\sum_{k \in N}\left(\theta_{k} \cdot x_{k}+y_{k}\right)}, \quad \forall i \in N .
$$

The nature of the contest might suggest that one of the investments should be treated as fixed for all contestants (say, $y_{i}=\overline{y_{i}}, \forall i \in N$ ) since it is not subject to choice during the contest. Then

$$
p_{i}(x)=\frac{\left(\alpha_{i}+\theta_{i} \cdot x_{i}\right)}{\sum_{k \in N}\left(\alpha_{k}+\theta_{k} \cdot x_{k}\right)}, \quad \forall i \in N,
$$

where $\alpha_{i}=\overline{y_{i}}$ for all $i \in N$. This is precisely the CSF used by Nti (2004). If we further assume that each contestant has the same level of the fixed investment, i.e., if $\overline{y_{i}}=\alpha$ for each $i \in N$, then

$$
p_{i}(x)=\frac{\left(\alpha+\theta_{i} \cdot x_{i}\right)}{\sum_{k \in N}\left(\alpha+\theta_{k} \cdot x_{k}\right)}, \quad \forall i \in N,
$$

which is the CSF used by Kolmar and Wagener (2005).

Example $2 f_{i}\left(x_{i}, y_{i}\right)=\left(x_{i}^{\theta_{i}} \cdot y_{i}^{\eta_{i}}\right)$, where $\theta_{i}>0, \eta_{i}>0$, and $\theta_{i}+\eta_{i}=\mu$, for all $i \in N$.

With this Cobb-Douglas production function, the CSF becomes

$$
p_{i}(x, y)=\frac{\left(x_{i}^{\theta_{i}} \cdot y_{i}^{\eta_{i}}\right)}{\sum_{k \in N}\left(x_{k}^{\theta_{k}} \cdot y_{k}^{\eta_{k}}\right)}, \quad \forall i \in N .
$$


If $y_{i}=\overline{y_{i}}$ for all $i \in N$, then

$$
p_{i}(x)=\frac{\left(\alpha_{i} \cdot x_{i}^{\theta_{i}}\right)}{\sum_{k \in N}\left(\alpha_{k} \cdot x_{k}^{\theta_{k}}\right)}, \quad \forall i \in N
$$

where $\alpha_{i}=\left(\overline{y_{i}}\right)^{\eta_{i}}$ for all $i \in N$. This CSF has been used by Cornes and Hartley (2005).

\subsection{Anonymity}

The CSF in Eq. (9) is not the non-anonymous Tullock-CSF axiomatized by Clark and Riis (1998) since $\theta_{i}$, the exponent of the choice variable $x_{i}$, can differ across contestants. It reflects the idea that different agents can possess different technologies for converting their actual investments into effective investments. Wars, elections, rent seeking, and sporting contests are best modeled by assuming that each agent possesses his own technology. We term such contests as unmediated contests. On the other hand, mediated contests are those wherein the technology of converting agents' actual investments into effective investments is determined by the contest administrator. The administrator may or may not use the same technology across contestants. We have introduced this distinction between mediated and unmediated contests to point out that the CSFs satisfying the following axiom of anonymity are better suited for analyzing mediated contests.

[A6] Anonymity $p_{i}(z)=p_{j}(z)$ if $z_{i}=z_{j}, \quad$ where $i \neq j, \quad$ and $i, j \in N$.

It can be easily verified that anonymity imposes the restriction that $f_{i}(\cdot)=f_{j}(\cdot)$ for all $i, j \in N$. Thus, a CSF satisfies axioms A1-A6 if and only if

$$
p_{i}(z)=\frac{f\left(z_{i}\right)}{\sum_{k \in N} f\left(z_{k}\right)}, \quad \forall z \in \mathbb{R}_{+}^{L N}, \quad \forall i \in N,
$$

where $f(\cdot)$ is increasing in each of its arguments. Recall that Clark and Riis (1998) drop the axiom of anonymity (with respect to the variable investment) to allow for the possibility that contestants with the same level of investment can have different winning probabilities as they can have different levels of contest-relevant personal characteristics. We would like to stress that since we interpret personal characteristics as a type of investment we do not need to drop the axiom of anonymity. Our framework thus helps clarify that imposing anonymity is actually equivalent to assuming that the technology of producing effective investments is same across all the contestants. We now illustrate the impact of anonymity by reconsidering the two examples presented above.

Example $1^{\prime} f\left(x_{i}, y_{i}\right)=\left(\theta \cdot x_{i}+y_{i}\right)$ for all $i \in N$, where $\theta>0$.

The CSF will be given by

$$
p_{i}(x, y)=\frac{\left(\theta \cdot x_{i}+y_{i}\right)}{\sum_{k \in N}\left(\theta \cdot x_{k}+y_{k}\right)}, \quad \forall i \in N .
$$


If $y_{i}=\overline{y_{i}}$ for all $i \in N$, then

$$
p_{i}(x)=\frac{\left(\alpha_{i}+x_{i}\right)}{\sum_{k \in N}\left(\alpha_{k}+x_{k}\right)}, \quad \forall i \in N,
$$

where $\alpha_{i}=\theta^{-1} \overline{y_{i}}$ for all $i \in N$. Following Corchon (2000), one can interpret $\alpha_{i}$ as the prior winning probability of contestant- $i$. If each contestant has the same level of the fixed investment, i.e., if $\overline{y_{i}}=c$ for each $i \in N$, then the above CSF becomes

$$
p_{i}(x)=\frac{\left(\alpha+x_{i}\right)}{\sum_{k \in N}\left(\alpha+x_{k}\right)}, \quad \forall i \in N,
$$

where the scalar $\alpha=\theta^{-1} c$ for all $i \in N .^{6}$

Example $2^{\prime} f\left(x_{i}, y_{i}\right)=\left(x_{i}^{\theta} \cdot y_{i}^{\eta}\right)$, for all $i \in N$, where $\theta>0, \eta>0$, and $\theta+\eta=\mu$. The CSF will be given by

$$
p_{i}(x, y)=\frac{\left(x_{i}^{\theta} \cdot y_{i}^{\eta}\right)}{\sum_{k \in N}\left(x_{k}^{\theta} \cdot y_{k}^{\eta}\right)}, \quad \forall i \in N
$$

If $y_{i}=\overline{y_{i}}$ for all $i \in N$, then

$$
p_{i}(x)=\frac{\left(\alpha_{i} \cdot x_{i}^{\theta}\right)}{\sum_{k \in N}\left(\alpha_{k} \cdot x_{k}^{\theta}\right)}, \quad \forall i \in N,
$$

where $\alpha_{i}=\left(\overline{y_{i}}\right)^{\eta}$ for all $i \in N$. This is the non-anonymous Tullock-CSF axiomatized by Clark and Riis (1998). The anonymous Tullock-CSF axiomatized by Skaperdas (1996) is obtained if we assume that the level of the fixed investment is the same for all contestants.

\section{Conclusion}

We consider contests in which each contestant can have multiple types of investments, some of which may be fixed. We distinguish between two types of contestsunmediated and mediated contests. In an unmediated contest each contestant uses his own technology (or, production function) to combine his actual investments and produce his effective investment; in a mediated contest the contest administrator chooses the technology for each contestant. The effective investments of all the contestants then determine the winning probabilities in both types of contests. We axiomatize

\footnotetext{
6 Amegashie (2006) uses the CSF given in Eq. (13) to model an unmediated contest (rent seeking) and interprets $\alpha$ as pure luck since each agent would be equally likely to win the contest if each $x_{i}$ is zero. It is not feasible to increase $\alpha$ for only one contestant while holding it fixed for the remaining contestants if $\alpha$ is interpreted as "pure" luck. We interpret this CSF as arising from a situation in which $\alpha_{i}$ can vary independently of $\alpha_{j}$, for any $i, j \in N$, but $\alpha_{i}$ happens to be the same for all $i \in N$.
} 
the family of CSFs satisfying homogeneity of order zero with respect to each type of investment of each contestant and some other basic axioms. The resulting family of CSFs is characterized by a homogeneous production function for each contestant, with the degree of homogeneity being the same for all contestants. We also clarify that the axiom of anonymity restricts the production function of effective investments to be identical for all contestants - an axiom that seems better suited to obtain CSFs in order to analyze mediated rather than unmediated contests.

\section{Appendix}

Proof of Theorem 1 This theorem is simply an extension of Theorem 1 in Skaperdas (1996) to the case where each agent can have multiple types of investments. Consistency and IIA $A_{S}$ imply

$$
\frac{p_{i}^{N}(z)}{p_{j}^{N}(z)}=\frac{p_{i}^{M}(z)}{p_{j}^{M}(z)}=\frac{p_{i}^{M}\left(z^{M}\right)}{p_{j}^{M}\left(z^{M}\right)} .
$$

Let $p_{i}^{N}(z)=[i]_{N}$ represent the probability that contestant- $i$ wins the global contest among all the $N$ contestants. Similarly, let $p_{i}^{M}(z)=p_{i}^{M}\left(z^{M}\right)=[i]_{M}$ represent the probability that contestant- $i$ wins the sub-contest among $M \subseteq N$ contestants. For the sub-contest between contestants in $M$,

$$
\sum_{i \in M}[i]_{M}=1
$$

This equality can be rewritten as

$$
\frac{[i]_{M}}{\sum_{k \neq i}^{k \in M}[k]_{M}} \cdot \frac{[j]_{M}}{[i]_{M}} \cdot \sum_{k \neq i}^{k \in M} \frac{[k]_{M}}{[j]_{M}}=1
$$

Consider the two player contests in which one player is always contestant- $j$. For such contests, let $[i]_{\{i, j\}}$ denote the probability that contestant $i$ wins it. By consistency

$$
\frac{[j]_{M}}{[i]_{M}}=\frac{[j]_{\{i, j\}}}{[i]_{\{i, j\}}}=\frac{1-[i]_{\{i, j\}}}{[i]_{\{i, j\}}}, \text { and } \quad \frac{[k]_{M}}{[j]_{M}}=\frac{[k]_{\{k, j\}}}{1-[k]_{\{k, j\}}} .
$$

Using the equalities in Eq. (19), Eq. (18) becomes

$$
\frac{[i]_{M}}{1-[i]_{M}} \cdot \frac{1-[i]_{\{i, j\}}}{[i]_{\{i, j\}}} \cdot \sum_{k \neq i}^{k \in M} \frac{[k]_{\{k, j\}}}{1-[k]_{\{k, j\}}}=1
$$

Let the investment vector of contestant- $j$ be fixed at $z_{0}$. It follows from IIA that we can define

$$
\frac{[i]_{\{i, j\}}}{1-[i]_{\{i, j\}}}=f_{i}\left(z_{i} ; z_{0}\right)=f_{i}\left(z_{i}\right), \quad \forall i \in M .
$$


Equation (20) can now be written as

$$
\frac{[i]_{M}}{1-[i]_{M}} \cdot \frac{1}{f_{i}\left(z_{i}\right)} \cdot \sum_{k \neq i}^{k \in M} f_{k}\left(z_{k}\right)=1
$$

Rearranging the above equation we obtain

$$
[i]_{M}=p_{i}^{M}\left(z^{M}\right)=\frac{f_{i}\left(z_{i}\right)}{\sum_{k \in M} f_{k}\left(z_{k}\right)}, \quad \forall i \in M, \forall M \subseteq N,
$$

which is the form given in Eq. (1). Finally, let $\tilde{z}=\left(\tilde{z}_{i}, \tilde{z}_{-i}\right)$ with $\tilde{z}_{i}>z_{i}$, but $\tilde{z}_{-i}=z_{-i}$. The axiom of marginal effects requires that

$$
p_{i}(\tilde{z})=p_{i}\left(\tilde{z}_{i}, z_{-i}\right)=\frac{f_{i}\left(\tilde{z}_{i}\right)}{f_{i}\left(\tilde{z}_{i}\right)+\sum_{k \in i \backslash M} f_{k}\left(z_{k}\right)}>\frac{f_{i}\left(z_{i}\right)}{\sum_{k \in M} f_{k}\left(z_{k}\right)}=p_{i}(\tilde{z})
$$

Rearranging the above equations gives

$$
f_{i}\left(\tilde{z}_{i}\right)>f_{i}\left(z_{i}\right)
$$

Hence, each $f_{i}(\cdot)$ must be an increasing function of each of its arguments.

Proof of Theorem 2 From Theorem 1,

$$
p_{i}^{N}(z)=\frac{f_{i}\left(z_{i}\right)}{\sum_{k \in N} f_{k}\left(z_{k}\right)}, \quad \forall i \in N
$$

We need to show that if $p_{i}(\lambda z)=p_{i}(z)$ for all $\lambda>0$, then

$$
\frac{f_{i}\left(\lambda z_{i}\right)}{f_{i}\left(z_{i}\right)}=\lambda^{\mu}, \quad \text { for all } \lambda>0, \quad \text { for all } i \in N,
$$

where $\mu$ is some strictly positive real number and is identical for each $i \in N$. Note that homogeneity implies

$$
p_{i}(\lambda z)=\frac{f_{i}\left(\lambda z_{i}\right)}{\sum_{k \in N} f_{k}\left(\lambda z_{k}\right)}=\frac{f_{i}\left(z_{i}\right)}{\sum_{k \in N} f_{k}\left(z_{k}\right)}=p_{i}(z) .
$$

The equalities above hold for all $i \in N$. Thus

$$
\frac{f_{i}\left(\lambda z_{i}\right)}{f_{i}\left(z_{i}\right)}=\frac{f_{j}\left(\lambda z_{j}\right)}{f_{j}\left(z_{j}\right)}=\frac{\sum_{k \in N} f_{k}\left(\lambda z_{k}\right)}{\sum_{k \in N} f_{k}\left(z_{k}\right)}, \quad \forall i, j \in N
$$

Since the last term in the above equality is the same for all $i \in N$, we can conclude that $\frac{f_{i}\left(\lambda z_{i}\right)}{f_{i}\left(z_{i}\right)}$ depends on $\lambda$ but not on $z_{i}$. Hence, if the vector of investments by contestant- $i$ 
were to be $\tilde{z_{i}} \in \mathbb{R}_{+}^{L}$, we would have

$$
\frac{f_{i}\left(\lambda z_{i}\right)}{f_{i}\left(z_{i}\right)}=\frac{f_{i}\left(\lambda \tilde{z_{i}}\right)}{f_{i}\left(\tilde{z_{i}}\right)}, \quad \text { for all } \lambda>0, \quad \text { for all } i \in N
$$

The above equation can be rewritten as

$$
\frac{f_{i}\left(\lambda z_{i}\right)}{f_{i}\left(\lambda \tilde{z_{i}}\right)}=\frac{f_{i}\left(z_{i}\right)}{f_{i}\left(\tilde{z_{i}}\right)}, \quad \text { for all } \lambda>0, \text { for all } i \in N
$$

Let us define

$$
g_{i}^{w}(\gamma)=f_{i}(\gamma w, \gamma)
$$

where $\gamma \in \mathbb{R}_{+}$and $w \in \mathbb{R}_{+}^{L-1}$.

For any strictly positive real numbers $\alpha$ and $\beta$

$$
\frac{g_{i}^{w}(\alpha \beta)}{g_{i}^{w}(\alpha)}=\frac{f_{i}(\alpha \beta w, \alpha \beta)}{f_{i}(\alpha w, \alpha)} .
$$

Using Eq. (30), the above equation (by treating $\alpha$ as $\lambda$, and then substituting $\alpha=1$ ) gives

$$
\frac{f_{i}(\alpha \beta w, \alpha \beta)}{f_{i}(\alpha w, \alpha)}=\frac{f_{i}(\beta w, \beta)}{f_{i}(w, 1)}=\frac{g_{i}^{w}(\beta)}{g_{i}^{w}(1)} .
$$

From Eq. (32) and (33) we get

$$
\frac{g_{i}^{w}(\alpha \beta)}{g_{i}^{w}(1)}=\frac{g_{i}^{w}(\alpha)}{g_{i}^{w}(1)} \cdot \frac{g_{i}^{w}(\beta)}{g_{i}^{w}(1)} .
$$

Equation (34) is one of Cauchy's fundamental functional equations (Aczel 1966) whose standard solution is given by

$$
g_{i}^{w}(\alpha)=g_{i}^{w}(1) \cdot \alpha^{h_{i}(w)}=f_{i}(w, 1) \cdot \alpha^{h_{i}(w)},
$$

where $h_{i}(\cdot)$ is some function of $w$. Let $\alpha w=z_{i}^{-l}=\left(z_{i}^{1}, \ldots, z_{i}^{l-1}, z_{i}^{l+1}, \ldots, z_{i}^{L}\right)$ and $\alpha=z_{i}^{l}$. This implies

$$
w=\frac{z_{i}^{-l}}{z_{i}^{l}}=\left(\frac{z_{i}^{1}}{z_{i}^{l}}, \ldots, \frac{z_{i}^{l-1}}{z_{i}^{l}}, \frac{z_{i}^{l+1}}{z_{i}^{l}}, \ldots, \frac{z_{i}^{L}}{z_{i}^{l}}\right)
$$

which in turn gives

$$
f_{i}(\alpha w, \alpha)=f_{i}\left(z_{i}^{-l}, z_{i}^{l}\right)=f_{i}\left(z_{i}\right)=f_{i}\left(\frac{z_{i}^{-l}}{z_{i}^{l}}, 1\right) \cdot\left(z_{i}^{l}\right)^{h_{i}\left(\frac{z_{i}^{-l}}{z_{i}^{l}}\right)} .
$$


The above equation implies

$$
\frac{f_{i}\left(\lambda z_{i}\right)}{f_{i}\left(z_{i}\right)}=\lambda^{h_{i}\left(\frac{z_{i}^{-l}}{z_{i}^{l}}\right)} .
$$

Since $\left(\frac{f_{i}\left(\lambda z_{i}\right)}{f_{i}\left(z_{i}\right)}\right)$ is the same for all $i \in N$, it is independent of $z_{i}$, and a function of $\lambda$ only. Hence, $h_{i}\left(\frac{z_{i}^{-l}}{z_{i}^{l}}\right)$ must be the same constant, say, $\mu$, for each $i \in N$. Moreover, $\mu$ must be a strictly positive real number for the axiom of marginal effects to be satisfied.

\section{References}

Aczel, J.: Lectures on Functional Equations and their Applications. New York: Academic Press (1966) Amegashie, J.A.: A contest success function with a tractable noise parameter. Public Choice 126, 135-144 (2006)

Clark, D.J., Riis, C.: Contest success functions: an extension. Econ Theory 11, 201-204 (1998)

Corchon, L.C.: On the allocative effects of rent seeking. J Public Econ Theory 2, 483-491 (2000)

Cornes, R., Hartley, R.: Asymmetric contests with general technologies. Econ Theory 26, 923-946 (2005)

Kolmar, M., Wagener, A.: Contests and the private provision of public goods. Working paper. Available at: http://homepage.univie.ac.at/Andreas.Wagener/papers/tenure.pdf (2005)

Nitzan, S.: Modelling rent seeking contests. Eur J Polit Econ 10, 41-60 (1994)

Nti, K.O.: Maximum efforts in contests with asymmetric valuations. Eur J Polit Econ 20, 1059-1066 (2004)

Rosen, S.: Prizes and incentives in elimination tournaments. Am Econ Rev 76, 701-715 (1986)

Skaperdas, S.: Contest success functions. Econ Theory 7, 283-290 (1996)

Skaperdas, S., Garfinkel, M.R.: Economics of conflict: an overview. In: Sandler T., Hartley, K. (eds.) Handbook of defense economics, vol. 2 (2006 to appear)

Snyder, J.M.: Election goals and the allocation of campaign resources. Econometrica 57, 637-660 (1989)

Szymanzki, S.: The economic design of sporting contests. J Econ Lit 61, 1137-1187 (2003) 